Acta Crystallographica Section D

Biological

Crystallography

ISSN 0907-4449

${ }^{\text {a}} \mathrm{F}$. Hoffmann-La Roche, Discovery Technologies, 4070 Basel, Switzerland, and bIsotechnika Inc., 5120 75th Street, Edmonton, Alberta T6E 6W2, Canada

₹ Current address: Hoffmann-La Roche, 340 Kingsland Street, Nutley, NJ 07110, USA.

Correspondence e-mail: michael.hennig@roche.com

\title{
Structural basis for the cyclophilin A binding affinity and immunosuppressive potency of E-ISA247 (voclosporin)
}

$E$-ISA247 (voclosporin) is a cyclosporin A analogue that is in late-stage clinical development for the treatment of autoimmune diseases and the prevention of organ graft rejection. The X-ray crystal structures of E-ISA247 and its stereoisomer $Z$-ISA247 bound to cyclophilin A have been determined and their binding affinities were measured to be 15 and $61 \mathrm{n} M$, respectively, by fluorescence spectroscopy. The higher affinity of $E$-ISA247 can be explained by superior van der Waals contacts between its unique side chain and cyclophilin A. Comparison with the known ternary structure including calcineurin suggests that the higher immunosuppressive efficacy of $E$-ISA247 relative to cyclosporin A could be a consequence of structural changes in calcineurin induced by the modified $E$-ISA247 side chain.

\section{Introduction}

Cyclosporin A (CsA) is a cyclic undecapeptide fungal metabolite that is used for the prevention of acute rejection in solid-organ transplantation (Graeb et al., 2004) and in the treatment of psoriasis (Sobell \& Hallas, 2003). CsA exerts its immunosuppressive activity in a heterodimeric complex with the peptidyl-prolyl cis-trans isomerase cyclophilin A (CypA; Hamawy, 2003). Once formed, this complex binds to and inhibits the calcium-dependent phosphatase calcineurin (Cn). This prevents dephosphorylation of the transcriptional activator nuclear factor of activated $\mathrm{T}$ cells and its translocation from the cytoplasm to the nucleus. Consequently, the production of many immunosignalling cytokines is blocked and the immune reaction is suppressed (Hamawy, 2003). However, the long-term use of CsA is limited by side effects, notably nephrotoxicity (Burdmann et al., 2003; Serkova et al., 2004; Zachariae et al., 1997).

The first X-ray structure of the CsA-CypA complex was reported at $2.8 \AA$ resolution from tetragonal crystals (Pflugl et al., 1993, 1994). Subsequently, CsA-CypA structures from orthorhombic (Mikol et al., 1993) and monoclinic (Ke et al., 1994) crystals that diffracted to $2.1 \AA$ resolution were published. In addition, several crystal structures of CypA complexed with different CsA derivatives and linear peptide ligands have been reported (Taylor et al., 1997). Finally, X-ray crystal structures of the ternary Cn-CypA-CsA complex were solved at 2.8 and $3.1 \AA$ resolution (Huai et al., 2002; Jin \& Harrison, 2002). Analysis of the CsA-Cn interface suggested that modification of the butenyl group of the first CsA residue 4-[(E)-2-butenyl]-4,N-dimethyl-L-threonine (Bmt1) might optimize its fit against the hydrophobic $\mathrm{Cn}$ surface and therefore increase the binding affinity.

In the continuous search for immunosuppressive compounds with improved efficacy and safety profiles, the racemic
Received 27 November 2010 Accepted 10 December 2010

PDB References: CypAE-ISA247, 3odi; CypAZ-ISA247, 3odl. 
CsA derivative ISA247 has been developed (Aspeslet et al., 2001). In the $E$ isomer of ISA247 ( $E$-ISA247; voclosporin) the native MeBmt1 (Fig. $1 a)$ is replaced by $4-[(2 E, 4 E Z)-2,4-$ pentadienyl]-4, $N$-dimethyl-L-threonine ( $E$-MePmt1; Fig. $1 b)$, while in the $Z$ isomer ( $Z$-ISA247) this residue is replaced by $\quad 4-[(2 Z, 4 E Z)-2,4-$ pentadienyl]-4, $N$-dimethyl-L-threonine (Z-MePmt1; Fig. 1c). E-ISA247 shows a greater immunosuppressive activity than CsA in vitro (Birsan et al.,2005) and in in vivo animal models of autoimmunity (Maksymowych et al., 2002) and transplantation (Aspeslet et al., 2001; Gregory et al., 2004). Two Phase III trials investigating the use of $E$-ISA247 for the treatment of moderate to severe psoriasis, a Phase IIb trial investigating the use of $E$-ISA247 for the prevention of organ graft rejection following kidney transplantation and two Phase II/III trials investigating the use of $E$-ISA247 for the treatment of non-infectious uveitis have been completed (Bissonnette et al., 2006; Dumont, 2004).

Here, we report the X-ray crystal structures of the CsA analogues $E$-ISA247 (voclosporin) and Z-ISA247 bound to CypA. The equilibrium dissociation constants of both CypAISA247 complexes are determined and are rationalized by the observed structural data. The impact of the stereoisomer configuration of E-ISA247 on the binding interaction with $\mathrm{Cn}$ and the resulting immunosuppressive activity are discussed.

\section{Materials and methods}

\subsection{Protein production}

The human CypA gene was inserted into a pET21a plasmid and overexpressed in Escherichia coli BL21 (DE3) cells. A 201 culture was grown at $303 \mathrm{~K}$ in LB medium and overexpression was induced at an optical density at $600 \mathrm{~nm}$ of 0.6 by adding IPTG to a final concentration of $0.5 \mathrm{mM}$. Cells were harvested after 6 h. $150 \mathrm{~g}$ biomass was resuspended in $750 \mathrm{ml}$ of a buffer consisting of $50 \mathrm{~m} M \mathrm{MES} \mathrm{pH} 6.5,10 \%$ saccharose, $5 \%$ glycerol, $10 \mathrm{mM} \mathrm{MgCl}$, $5 \mathrm{~m} M$ TCEP, $0.02 \% \mathrm{NaN}_{3}, 30 \mathrm{mg} \mathrm{l}^{-1}$ DNase I and four tablets of Complete protease-inhibitor cocktail (F. Hoffmann-La Roche) per litre. The cells were disrupted, the lysate was microfiltrated $(0.2 \mu \mathrm{m})$ and concentrated by ultrafiltration $(5 \mathrm{kDa})$ to about $200 \mathrm{ml}$ and the buffer was exchanged by diafiltration to $20 \mathrm{~m} M$ sodium phosphate $\mathrm{pH}$ 6.25, $2 \mathrm{mM}$ TCEP, 5\% glycerol, $0.02 \% \quad \mathrm{NaN}_{3}$ with four tablets of Complete protease-inhibitor cocktail 
Table 1

Data-collection and refinement statistics.

Values in parentheses are for the highest resolution shell.

\begin{tabular}{|c|c|c|}
\hline & $E$-ISA247 & $Z$-ISA247 \\
\hline PDB code & 3odi & 3odl \\
\hline Space group & $P 2_{1}$ & $P 2_{1} 2_{1} 2_{1}$ \\
\hline Unit-cell parameters $\left(\AA \AA^{\circ}\right)$ & $\begin{array}{c}a=69.2, b=161.3 \\
c=93.7 \\
\alpha=\gamma=90.0 \\
\beta=100.1\end{array}$ & $\begin{array}{l}a=132.4, b=141.1, \\
\quad c=149.1, \\
\alpha=\beta=\gamma=90.0\end{array}$ \\
\hline Copies per ASU† & 10 & 10 \\
\hline X-ray source & $\mathrm{Cu} K \alpha$ & SLS X06SA \\
\hline Wavelength $(\AA)$ & 1.5418 & 0.9799 \\
\hline Resolution range $(\AA)$ & $25.0-2.2(2.28-2.20)$ & $40.0-2.3(2.38-2.30)$ \\
\hline No. of unique reflections & 93907 (9188) & 123151 (12158) \\
\hline Completeness (\%) & $91.8(88.0)$ & $100.0(100.0)$ \\
\hline Multiplicity & $3.3(3.1)$ & $9.5(9.4)$ \\
\hline Mean $I / \sigma(I)$ & $13.3(2.6)$ & $22.1(6.7)$ \\
\hline$R_{\text {merge }} \neq$ & $0.082(0.399)$ & $0.113(0.424)$ \\
\hline$B$ factor from Wilson plot $\left(\AA^{2}\right)$ & 31.0 & 30.5 \\
\hline$R_{\text {cryst }}$ & 0.164 & 0.169 \\
\hline$R_{\text {free }}(\%)$ & 0.220 & 0.207 \\
\hline \multicolumn{3}{|l|}{ Root-mean-square deviations } \\
\hline Bond lengths $(\AA)$ & 0.008 & 0.007 \\
\hline Bond angles $\left({ }^{\circ}\right)$ & 1.09 & 1.05 \\
\hline No. of protein residues/atoms & $1644 / 12611$ & $1650 / 12660$ \\
\hline No. of ligand residues/atoms & $110 / 860$ & $110 / 860$ \\
\hline No. of water molecules & 1510 & 1726 \\
\hline \multicolumn{3}{|l|}{ Average $B$ factor $\left(\AA^{2}\right)$} \\
\hline All atoms & 36.4 & 24.4 \\
\hline Water molecules & 44.6 & 34.4 \\
\hline \multicolumn{3}{|c|}{ Ramachandran plot statistics, residues in (\%) } \\
\hline Favoured regions & 96.2 & 96.4 \\
\hline Allowed regions & 3.8 & 3.6 \\
\hline Outlier regions & 0.1 & 0.0 \\
\hline
\end{tabular}

$\dagger$ Number of CypA-ISA247 complexes per asymmetric unit in the crystal. $\ddagger R_{\text {merge }}=$ $\sum_{h k l} \sum_{i}\left|I_{i}(h k l)-\langle I(h k l)\rangle\right| / \sum_{h k l} \sum_{i} I_{i}(h k l)$.

(F. Hoffmann-La Roche) per litre. The protein solution was loaded onto a Macro-Prep Ceramic Hydroxyapatite Type I $20 \mu \mathrm{m}$ column (Bio-Rad) and eluted with a gradient from 20 to $500 \mathrm{~m} M$ sodium phosphate. The pooled CypA fractions were dialysed against $20 \mathrm{~m} M$ MES $\mathrm{pH}$ 6.3, $1.5 \mathrm{~m} M$ TCEP, $0.02 \%$ $\mathrm{NaN}_{3}$, loaded onto a Source 15S column (Amersham Biosciences) and eluted with an $\mathrm{NaCl}$ gradient. Finally, CypA was purified on a Superdex 75 Prep-Grade (Amersham Biosciences) size-exclusion column equilibrated with $10 \mathrm{mM}$ sodium/potassium phosphate $\mathrm{pH} 6.0,100 \mathrm{~m} M \mathrm{NaCl}, 2 \mathrm{~m} M$ TCEP, $0.02 \% \mathrm{NaN}_{3}$.

\subsection{Crystallization, $X$-ray data collection and model refinement}

For CypA-ISA247 complex formation, CypA was concentrated to $50 \mathrm{mg} \mathrm{ml}^{-1}$ and mixed with $2 \mathrm{mM} \mathrm{E-ISA247} \mathrm{or}$ $Z$-ISA247. The solution was incubated for $30 \mathrm{~min}$ at room temperature followed by $24 \mathrm{~h}$ on ice. The protein-peptide complexes were crystallized in hanging drops by vapour diffusion at $295 \mathrm{~K}$ using the same precipitant solution consisting of 20\%(w/v) PEG 3350, $100 \mathrm{~m} M$ MES pH 6.5 and $200 \mathrm{~m} M$ ammonium sulfate. For X-ray data collection at $100 \mathrm{~K}$, the crystals were transferred to precipitant solution containing an additional $20 \%$ glycerol and flash-frozen with liquid nitrogen. X-ray diffraction images of a CypA-E-ISA247 complex crystal were collected on a MAR345 image-plate detector (MAR Research) using $\mathrm{Cu} K \alpha$ radiation from a Nonius FR591 rotating-anode X-ray generator. Images for CypA-Z-ISA247 crystals were collected on a $165 \mathrm{~mm}$ MAR CCD detector (MAR Research) using synchrotron radiation on the Swiss Light Source X06SA beamline. CypA-E-ISA247 complex data were processed with the $H K L$ program suite (Otwinowski \& Minor, 1997) to $2.2 \AA$ resolution in space group $P 2_{1}$ and CypA-Z-ISA247 data were processed to $2.3 \AA$ resolution in space group $P 2_{1} 2_{1} 2_{1}$ (see Table 1 for statistics). Molecular-replacement solutions were obtained with the program MOLREP (Vagin \& Teplyakov, 2010) using the CypA-CsA crystal structure (PDB entry 2rma; Ke et al., 1994) as a search model. Ten CypA-inhibitor complexes were identified in both space groups. Initial $\left|F_{\mathrm{o}}\right|-\left|F_{\mathrm{c}}\right|$ maps calculated after rigid-body refinement against the CypA-E-ISA247 and CypA-Z-ISA247 data clearly showed the additional C atom of MePmt1 and the difference between its $E$ and $Z$ isomers. Both structures were refined by manual model fitting using the graphics program MOLOC (Gerber, 1992), model refinement using REFMAC5 (Murshudov et al., 1997) and the placement of water molecules with $A R P / w A R P$ (Perrakis et al., 1997). $B$ factors were not restrained. $5 \%$ of the diffraction data were excluded from the refinement process for crossvalidation. In the final models, the ten CypA-inhibitor complexes in the respective asymmetric units are essentially identical. The refinement statistics are summarized in Table 1. In the CypA-E-ISA247 structure Gly14 of chain $M$ is poorly defined in the electron-density maps and is the only Ramachandran plot outlier. The coordinates and structure factors of the CypA-E-ISA247 and CypA-Z-ISA247 structures have been deposited in the Protein Data Bank with PDB codes 3odi and 3odl, respectively.

\subsection{Fluorescence spectroscopy}

Fluorescence measurements were performed on an SLMAminco 8100 double-grating spectrofluorometer using pre-

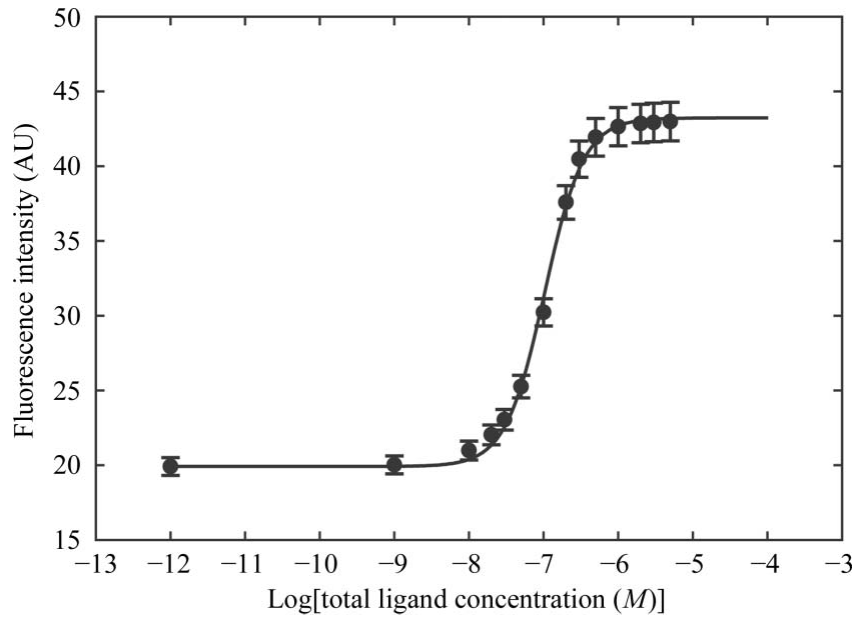

Figure 2

Fluorescence titration curve of the E-ISA247 isomer. The measured fluorescence intensities after correction for protein dilution (dots) are fitted with a sigmoidal curve (line). 
viously described methods (Huber et al., 2004). The intrinsic fluorescence of CypA was excited through its single tryptophan at $280 \mathrm{~nm}$ and was detected at $340 \mathrm{~nm}$. The equilibrium dissociation constant $K_{\mathrm{d}}$ was determined by fluorescence titration at $200 \mathrm{n} M$ protein concentration in $10 \mathrm{~m} M$ HEPES pH 7.4, $150 \mathrm{~m} M \mathrm{NaCl}$. Aliquots of $E$-ISA247, Z-ISA247 and CsA in DMSO were added to the protein solution $(2 \mathrm{ml})$ and the resulting fluorescence intensities were measured. The final and constant DMSO concentration was $1.7 \%$. The ISA247 isomers do not absorb at $280 \mathrm{~nm}$ and thus do not fluoresce. The measured fluorescence intensities were corrected for protein dilution (Birdsall et al., 1983). A plot of the corrected fluorescence intensity versus ligand concentration was fitted with a sigmoidal curve (Fig. 2), from which the $K_{\mathrm{d}}$ was computed according to the law of mass action for a one-to-one protein-ligand complex (Eftink, 1997). The reported $K_{\mathrm{d}}$ values are averages from three titrations.

\section{Results and discussion}

\subsection{ISA247 isomers do not induce major structural changes in the drug-CypA complex}

The structures of CypA complexed with E-ISA247 and Z-ISA247 were determined at 2.2 and $2.3 \AA$ resolution, respectively, with excellent quality of the electron density. In both complexes, the structure of CypA, the backbone conformation of the ISA247 isomers and the nonsubstituted ISA247 side chains $2-11$ are essentially the same as in the structure of the CypA-CsA complex (Ke et al., 1994). Significant differences between the three CypA-ligand structures are only observed in the side-chain conformation of residue 1 of the undecapeptides (Figs. $1 f-1 h$ ). The calculated electrondensity maps clearly show the elongated shape of the MePmt1 $E$ isomer (Fig. 1d) and the bent shape of the $Z$ isomer (Fig. 1e). We conclude that the chemical modifications of the ISA247 isomers at residue 1 (Figs. $1 a-1 c$ ) do not induce major rearrangements of the CypA side chains in the drug-CypA complex and that functional differences between the two ISA247 isomers result from the structural difference at the modified MePmt1 residue only.

\subsection{ISA247 stereoisomers differ in their interaction with CypA}

We have determined the binding affinities of E-ISA247, Z-ISA247 and CsA to its pharmacological binding partner CypA using fluorescence spectroscopy. The affinity of $E$-ISA247, with a $K_{\mathrm{d}}$ value of $15 \pm 4 \mathrm{n} M$, is essentially the same as the $K_{\mathrm{d}}$ of $13 \pm 4 \mathrm{n} M$ found for CsA. Z-ISA247, however, binds to CypA with an approximately fourfold higher dissociation constant $\left(K_{\mathrm{d}}=61 \pm 9 \mathrm{n} M\right)$. This further strengthens the decision made early in the development of voclosporin to focus on the $E$ isomer of ISA247 rather than to progress with the easier-to-produce racemate.

The only significant difference in the crystal structures of CypA complexed with Z-ISA247, E-ISA247 and CsA is observed in the side-chain conformation of the undecapeptide residue 1 (Figs. $1 f-1 h$ ). In the CypA-CsA complex MeBmt1 is positioned at the calcineurin-binding composite surface, between the CsA residues N-methylated leucine (MeLeu) 4, MeLeu6 and MeLeu10 on one side and the CypA residues Asn102, Ala103, Gly104 and His126 on the other side (Fig. 1f). The MeBmt1 side chain is in van der Waals contact with the Ala103 backbone atoms. In the crystal structures of $E$-ISA247 (Fig. $1 g$ ) and Z-ISA247 (Fig. $1 h$ ) bound to CypA, the interaction patterns between the modified MePmt1 side chains and the Ala103 backbone differ from the pattern observed in the CypA-CsA structure (Fig. 1f). While E-MePmt1 and MeBmt1 demonstrate high van der Waals complementarity with CypA, $Z$-MePmt leaves a gap between the protein and Z-ISA247. This is reflected in the distances of the CypA Ala103 carbonyl groups to the MeBmt1 (4.0 $), E$-MePmt1 $(3.5 \AA)$ and $Z$-MePmt $(5.0 \AA) \mathrm{CZ}$ atoms in the respective structures. We suggest that lack of surface complementarity between CypA and $Z$-MePmt is the reason for the reduced affinity of the CsA analogue $Z$-ISA247.

\subsection{Impact of MeBmt1 modifications on $\mathrm{Cn}$ inhibition}

CsA binds to CypA and this heterodimeric complex binds to and inhibits calcineurin, inducing immunosuppression. The X-ray crystal structures of the ternary Cn-CypA-CsA complex show that the binary CypA-CsA complex binds to a composite surface made up of residues from the $\mathrm{Cn}$ catalytic and regulatory subunits (Huai et al., 2002; Jin \& Harrison, 2002). In many cases the biological activity of CsA derivatives correlate with their affinity for CypA, but notable exceptions have been reported (Kallen et al., 1998). In general, modifications at the CypA-binding residues 1, 2, 9, 10 or 11 that

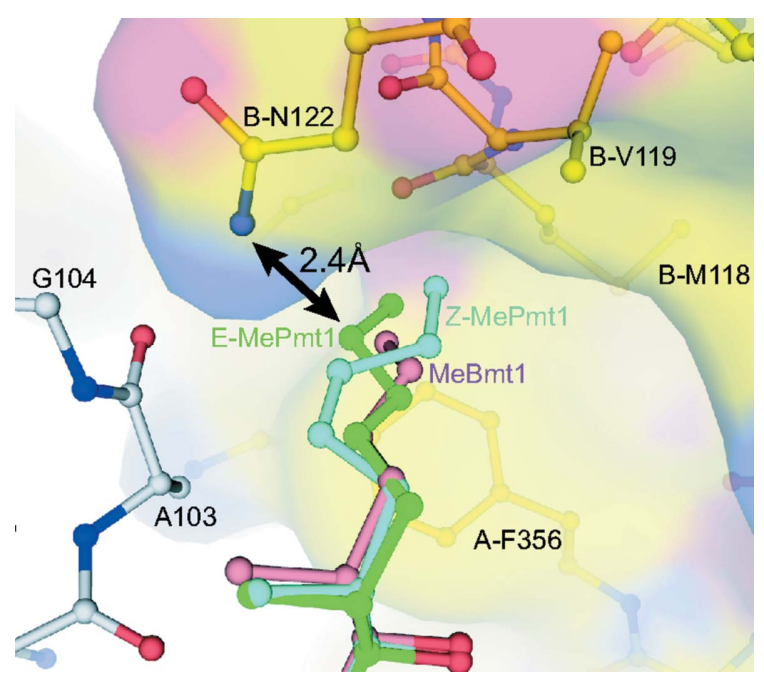

Figure 3

Superposition of the ternary Cn-CypA-CsA complex with the binary CypA-ISA247 complexes. Cn, CypA and the CsA residue MeBmt1 of the ternary complex (PDB entry 1m63; Huai et al., 2002) are displayed in yellow, grey and pink, respectively. The MePmt1 residues of $E$-ISA247 and $Z$-ISA247 are shown in green and cyan, respectively. A close contact between $E$-ISA247 and Cn (carbon-to-nitrogen distance of $2.4 \AA$ ) is marked by a black arrow. 
diminish binding to CypA also result in diminished immunosuppressive activity, while modifications of the effector-loop residues 4,5 or 6 can strongly affect the immunosuppressive activity without significantly affecting CypA binding (Kallen et al., 1998; Taylor et al., 1997). E-ISA247 exemplifies an exception to this rule: it shows affinity for CypA comparable with that of CsA but superior immunosuppressive activity (Birsan et al., 2005). To analyze the structural basis for this observation, we superimposed the CypA $\mathrm{C}^{\alpha}$ atoms of the binary CypA-ISA247 complexes with the CypA $\mathrm{C}^{\alpha}$ atoms of the ternary Cn-CypA-CsA complex and analysed the ISA247-Cn interfaces of the resulting Cn-CypA-ISA247 structure models (Fig. 3). Interestingly, the interaction patterns between Cn and the ISA247 isomers differ markedly from that observed with CsA. In the E-ISA247 ternarycomplex superposition model the distance between the side chains of $E$-MePmt 1 and the Cn residue Asn 122 is only $2.4 \AA$. This demonstrates that at least one of these two amino acids has to adopt a significantly different conformation in order to avoid repulsion. Very limited conformational flexibility has been observed for the MeBmt1 side chain of CsA in the published CypA complex crystal structures, indicating that the $\mathrm{Cn}$ residue Asn122 is more likely to be affected. The modification of the first residue of $E$-ISA247 is unlikely to significantly change its conformational flexibility, in contrast to the modification of the fourth residue of the CsA analogue Debio 025 , which not only limits the number of available side-chain conformations but also prevents binding of the Debio 025CypA complex to Cn (Landrieu et al., 2010). Asn122 is part of the so-called 'latch region' of the Cn regulatory subunit (Huai et al., 2002; Jin \& Harrison, 2002). This loop region is involved in activation of the Cn catalytic subunit and amino-acid substitutions within this loop induce varying degrees of CsA resistance (Milan et al., 1994). It has been suggested that modulation of Cn activity by CypA-CsA might be not only a consequence of spatial blockade of protein substrates but also of a direct involvement of CypA-CsA in the regulation of $\mathrm{Cn}$ activity (Huai et al., 2002). This would provide one possible explanation for the differential immunosuppressive activities of the equipotent CypA binders E-ISA247 and CsA. Another possible explanation would be that the CypA-E-ISA247 complex binds to $\mathrm{Cn}$ with higher affinity as a result of the predicted structural changes at the Cn-E-ISA247 interface around residue E-MePmt1.

We thank Agnes Weiss and Beat Wipf for providing fermentation support and Clemens Schulze-Briese and Takashi Tomizaki (Swiss Light Source, Villigen, Switzerland) for support at the X06SA synchrotron beamline.

\section{References}

Aspeslet, L., Freitag, D., Trepanier, D., Abel, M., Naicker, S., Kneteman, N., Foster, R. \& Yatscoff, R. (2001). Transplant. Proc. 33, 1048-1051.

Birdsall, B., King, R. W., Wheeler, M. R., Lewis, C. A. Jr, Goode, S. R., Dunlap, R. B. \& Roberts, G. C. (1983). Anal. Biochem. 132, 353-361.

Birsan, T., Dambrin, C., Freitag, D. G., Yatscoff, R. W. \& Morris, R. E. (2005). Transpl. Int. 17, 767-771.

Bissonnette, R., Papp, K., Poulin, Y., Lauzon, G., Aspeslet, L., Huizinga, R., Mayo, P., Foster, R. T., Yatscoff, R. W. \& Maksymowych, W. P. (2006). J. Am. Acad. Dermatol. 54, 472-478.

Burdmann, E. A., Andoh, T. F., Yu, L. \& Bennett, W. M. (2003). Semin. Nephrol. 23, 465-476.

Dumont, F. J. (2004). Curr. Opin. Investig. Drugs, 5, 542-550.

Eftink, M. R. (1997). Methods Enzymol. 278, 221-257.

Gerber, P. R. (1992). Biopolymers, 32, 1003-1017.

Graeb, C., Arbogast, H., Guba, M., Jauch, K. W. \& Land, W. (2004). Transplant. Proc. 36, 125-129.

Gregory, C. R., Kyles, A. E., Bernsteen, L., Wagner, G. S., Tarantal, A. F., Christe, K. L., Brignolo, L., Spinner, A., Griffey, S. M., Paniagua, R. T., Hubble, R. W., Borie, D. C. \& Morris, R. E. (2004). Transplantation, 78, 681-685.

Hamawy, M. M. (2003). Drug News Perspect. 16, 277-282.

Huai, Q., Kim, H.-Y., Liu, Y., Zhao, Y., Mondragon, A., Liu, J. O. \& Ke, H. (2002). Proc. Natl Acad. Sci. USA, 99, 12037-12042.

Huber, W., Perspicace, S., Kohler, J., Müller, F. \& Schlatter, D. (2004). Anal. Biochem. 333, 280-288.

Jin, L. \& Harrison, S. C. (2002). Proc. Natl Acad. Sci. USA, 99, $13522-$ 13526.

Kallen, J., Mikol, V., Taylor, P. \& Walkinshaw, M. D. (1998). J. Mol. Biol. 283, 435-449.

Ke, H., Mayrose, D., Belshaw, P. J., Alberg, D. G., Schreiber, S. L., Chang, Z. Y., Etzkorn, F. A., Ho, S. \& Walsh, C. T. (1994). Structure, 2, 33-44.

Landrieu, I., Hanoulle, X., Bonachera, F., Hamel, A., Sibille, N., Yin, Y., Wieruszeski, J. M., Horvath, D., Wei, Q., Vuagniaux, G. \& Lippens, G. (2010). Biochemistry, 49, 4679-4686.

Maksymowych, W. P., Jhangri, G. S., Aspeslet, L., Abel, M. D., Trepanier, D. J., Naicker, S., Freitag, D. G., Cooper, B. L., Foster, R. T. \& Yatscoff, R. W. (2002). J. Rheumatol. 29, 1646-1652.

Mikol, V., Kallen, J., Pflugl, G. \& Walkinshaw, M. D. (1993). J. Mol. Biol. 234, 1119-1130.

Milan, D., Griffith, J., Su, M., Price, E. R. \& McKeon, F. (1994). Cell, 79, 437-447.

Murshudov, G. N., Vagin, A. A. \& Dodson, E. J. (1997). Acta Cryst. D53, 240-255.

Otwinowski, Z. \& Minor, W. (1997). Methods Enzymol. 276, 307-326.

Perrakis, A., Sixma, T. K., Wilson, K. S. \& Lamzin, V. S. (1997). Acta Cryst. D53, 448-455.

Pflugl, G., Kallen, J., Jansonius, J. N. \& Walkinshaw, M. D. (1994). J. Mol. Biol. 244, 385-409.

Pflugl, G., Kallen, J., Schirmer, T., Jansonius, J. N., Zurini, M. G. \& Walkinshaw, M. D. (1993). Nature (London), 361, 91-94.

Serkova, N. J., Christians, U. \& Benet, L. Z. (2004). Mol. Interv. 4, 97-107.

Sobell, J. M. \& Hallas, S. J. (2003). Semin. Cutan. Med. Surg. 22, 187-195.

Taylor, P., Husi, H., Kontopidis, G. \& Walkinshaw, M. D. (1997). Prog. Biophys. Mol. Biol. 67, 155-181.

Vagin, A. \& Teplyakov, A. (2010). Acta Cryst. D66, 22-25.

Zachariae, H., Kragballe, K., Hansen, H. E., Marcussen, N. \& Olsen, S. (1997). Br. J. Dermatol. 136, 531-535. 